\title{
sciendo
}

\section{Validity of Performance Assessment Methods in Elite Paracanoeists}

\author{
by \\ Michal Starczewski ${ }^{1}$, Piotr Zmijewski², Andrzej Klusiewicz ${ }^{3}$
}

\begin{abstract}
The problem of valid and reliable research methods in paralympic sports seems of great significance, thus the aim of this study was to evaluate the validity of the Wingate test (WAnT) in assessment of anaerobic performance in elite paracanoeists competing in $200 \mathrm{~m}$ races. Seven paralympic athletes (32.6 \pm 7.76 years), medal winners and finalists of the Paralympic Games, World and European Championships, as well as World Cups were examined. The evaluations were conducted during a competition which was held at the paralympic distance of $200 \mathrm{~m}$, and included the WAnT for upper limbs and the anaerobic threshold test (ATT). Correlations of selected indices of mechanical work and power in the WAnT, as well as anaerobic threshold (AT) power, with the time recorded during the competition were used to determine indices which affected paracanoeing performance. The results indicated strong negative correlations with time achieved in the competition between peak power and total work in the WAnT, and AT power $(R=-0.796 p=$ $0.032 ; R=-0.882 p=0.009 ; R=-0.900 p<0.001$, respectively). The research confirmed the validity of the WAnT for the upper limbs to assess the performance level of paracanoeists. It may be concluded that the WAnT and ATT may be used for monitoring performance during the paralympic $200 \mathrm{~m}$ race.
\end{abstract}

Key words: paracanoe $200 \mathrm{~m}$ race, testing, anaerobic performance, prediction.

\section{Introduction}

Paracanoe is one of the most recent sports practiced within canoeing. The international debut of the sport took place during the 2009 Canoe Sprint World Championships in Dartmouth, Nova Scotia, Canada. Since then, the sport has developed dynamically, and in 2012, it was included in the program of the Paralympic Games. In 2016, paralympic races were held in Rio (Starczewski, 2013). Events of this sport include kayak single (K1) and canoe single (V1) races at a distance of $200 \mathrm{~m}$. There are three classifications used in both $\mathrm{K} 1$ and V1, to which competitors are assigned according to the type of disability (International Canoe Federation, 2019; Starczewski, 2013).

Considering the duration of exercise typical for paracanoeing sports (lasting from 38 to $65 \mathrm{~s}$ ), it can be assumed that anaerobic metabolism prevails during such efforts. This is confirmed by the research in the group of able-bodied canoeists, in whom the competitive effort over a distance of $250 \mathrm{~m}$ lasted aproximatley $62 \mathrm{~s}$ and the demand of anaerobic metabolism was 59.5\% (Zamparo et al., 1999). A study of the US national team during a simulated $200 \mathrm{~m}$ race on a canoe ergometer found that the input of aerobic processes in this kind of exercise was $37 \%$, which seems to confirm the above presented thesis (Byrnes and Kearney, 1997).

Given the significant contribution of anaerobic metabolism to the energy demand at the paracanoeing racing distances, it is advisable to implement the Wingate upper limb (WAnT) test to evaluate anaerobic capacity of these disabled athletes. This tool is commonly used for

1 - Faculty of Rehabilitation, Jozef Pilsudski University of Physical Education in Warsaw, Warsaw, Poland.

2 - Jozef Pilsudski University of Physical Education in Warsaw, Warsaw, Poland

3 - Faculty of Physical Education and Health, Biala Podlaska, Poland, Jozef Pilsudski University of Physical Education in Warsaw 
research in sports where the driving force comes from the upper limbs, both among able-bodied and disabled athletes (Byrnes and Kearney, 1997; Hutzler et al., 1998; Sitkowski and Grucza, 2009; Someren and Palmer, 2003). The research of elite UK canoeists provided interesting insights into the relationship demonstrated between the $200 \mathrm{~m}$ results and anaerobic capacity variables, especially total work performed in the WAnT (Someren and Palmer, 2003). Furthermore, it was found that the above-mentioned indices discriminated between national and international level athltes. This observation leads to the conclusion that the WAnT may also be reliable in the assessment of exercise capacity of paracanoe athletes. In previous studies of disabled Polish canoeists, the WAnT for upper limbs was used to classify athletes into particular performance groups (Starczewski, 2014). To the best of our knowledge, although the literature on the diagnostic value of the anaerobic threshold (AT) in canoeing is rather extensive (Bishop, 2000; Van Someren and Howatson, 2008), no attempts have been made to determine the relationship between $200 \mathrm{~m}$ race performance of disabled canoeists and the anaerobic capacity and AT variables.

Therefore, the present study aimed to evaluate the validity of the WAnT for upper limbs. Secondly, we attempted to indirectly assess the performance level of paracanoeists competing in $200 \mathrm{~m}$ races using the WAnT and AT indices.

\section{Methods}

\section{Participants}

Seven athletes (two females and five males) participated in the study (32.6 \pm 7.76 years of age, $70.3 \pm 12.2 \mathrm{~kg}$ of body mass and $4.69 \pm 2.84$ years of sports experience). They constituted a representative group of Polish paracanoeists (ca. $70 \%$ of the total number of athltes in 2016). They were medalists of the Paralympic Games and World Championships. Four of participants had spinal cord injuries or diseases, and three of them lower legs impairment.

Due to the small size of the study group resulting from the specific nature of disabled sports, our analyses did not include a division according to sex. It should be emphasized that according to research conducted on able-bodied wrestlers, the amount of energy supplied from glycolysis does not depend on sex, which justifies the inclusion of both male and female athletes into one study group (Hübner-Wozniak et al., 2004).

\section{Design and Procedures}

The research protocol was approved by the local Ethics Committee in compliance with the Declaration of Helsinki. All tests were performed on two consecutive days at the same time of the day (between $10 \mathrm{am}$ and $1 \mathrm{pm}$ ). On the first day, $200 \mathrm{~m}$ races were held on-water. On the second day, testing was performed in laboratory settings. First the WAnTs were completed, and after a 1-h passive recovery period, the anaerobic threshold tests (ATTs) were conducted. Athletes were instructed not to perform intense exercise on the day before testing, and to consume their last meal at least $2 \mathrm{~h}$ before the scheduled test (Figure 1).

\section{On-water test (OWT)}

Race results were obtained during the qualifying competition for the Polish paracanoe national team. Individual $200 \mathrm{~m}$ races were used to evaluate the current sports performance level. During the competition photocells were used for timing.

\section{Exercise tests}

The $30 \mathrm{~s}$ Wingate Anaerobic capacity test (WAnT) was performed on a Monark ergocycle (Monark Exercise $A B$, Sweden), modified for upper limb tests in a sitting or a standing position, equipped with MCE v. 5.2 software ("JBA", Poland). The sampling rate was $1000 \mathrm{~Hz}$. The test was preceded by a 5-min warm-up which included up to three accelerations. A 5min passive rest was allowed between the warmup and the following testing. During the WAnT, modified loads were used: for women $4 \%$ of body weight for KL1 and KL2 (kayak level 1 and 2 sport classes) classifications and 5\% of body weight for KL3, while for men, the load was $5 \%$ of body weight for KL1 and KL2 and $6.2 \%$ of body weight for KL3. The method of choosing the exercise load was consistent with earlier reports on anaerobic capacity tests in disabled athletes (Dotan and Bar-Or, 1983; Starczewski and Molik, 2014).

\section{Anaerobic threshold test (ATT)}

A submaximal graded exercise test was performed on the Dansprint Pro air-braked kayak ergometer (Dansprint ApS, Denmark). The 
test consisted of five 4-min exercise bouts separated by $1 \mathrm{~min}$ rest intervals for blood sampling. The test loads were $0.3+0.35 \mathrm{~W} / \mathrm{kg}$ for men in classes KL1 and KL2, $0.3+0.5 \mathrm{~W} / \mathrm{kg}$ in $\mathrm{KL3}$, while for women, these values were $0.2+$ $0.3 \mathrm{~W} / \mathrm{kg}$ and $0.2+0.4 \mathrm{~W} / \mathrm{kg}$, respectively. The AT was determined by interpolation of recorded values of exercise indices for blood lactate concentration corresponding to $4 \mathrm{mmol} / \mathrm{l}$.

To assess validity of the WAnT for paracanoeists in the third minute after the WAnT and the OWT blood was drawn from the earlobe to determine blood lactate concentration (LA). The Super GL2 dr Müller analyzer was used (Dr. Müller Gerätebau GmbH, Germany). The measurement error of the method is less than $1.5 \%$.

\section{Statistical Analysis}

The results are presented as means and standard deviations. The Shapiro-Wilk test was used to test variables for normal distribution. The Student's t-test was performed to assess the difference in the metabolic response (lactate levels) to the OWT and the WAnT. The BlandAltman plot was prepared to compare the results of both tests. Pearson's correlations were calculated to determine the relationships of chosen anaerobic indices with sports performance in paracanoeing (WAnT and ATT were correlated with $200 \mathrm{~m}$ results). The statistical significance was set at $p<0.05$. All calculations were performed with STATISTICA software (v. 12.0, StatSoft, USA).

\section{Results}

Metabolic responses to different types of effort, which included the $200 \mathrm{~m}$ race and the $30 \mathrm{~s}$ WAnT for upper limbs were compared. The mean LA concentration in the $3^{\text {rd }}$ min of recovery was $8.27 \pm 2.20 \mathrm{mmol} / \mathrm{l}$, whereas after the Wingate test it reached $8.57 \pm 2.18 \mathrm{mmol} / \mathrm{l}$. The results of the Student's t-test showed no significant differences $(p=0.42)$ in the metabolic response expressed in blood lactate cooncentrations following both tests (Figure 2).

Furthermore, there was a significant correlation between LA concentration from previously mentioned tests, which reached a value of $\mathrm{R}=0.913(p=0.004)$ (Figure 3). According to Rosenthal (1996), such a result indicates a very strong effect size between the variables studied.
A Bland-Altman plot was used to illustrate the bias between LA concentration after the OWT and the WAnT. Bias effect size scores were trivial for OWT LA concentration (-0.294) in relation to the WAnT. The Bland-Altman statistics showed that the WAnT LA concentration was accurate for performance assessment of on-water LA concentration (Figure 4).

Table 2 shows a summary of the Pearson's correlation results of the time achieved in the $200 \mathrm{~m}$ race and the indices measured in the WAnT and ATT. Indices which showed a significant relationship with sports performance in the anaerobic capacity test were total work $(W)$, mean power (Pmean), and peak power $\left(P_{\text {peak }}\right)$, as well as anaerobic threshold power (PАт). No significant relationships were found for the time to reach peak power, time of maintaining peak power and the fatigue index in the WAnT (Table1).

\section{Discussion}

The main finding of the study was that there were no significant differences in the metabolic response expressed in LA levels following the $200 \mathrm{~m}$ race and the WAnT for the upper limbs. This observation seems significant for research and coaching practice in paracanoeing. The lack of difference in LA levels may indicate the validity of the WAnT for the evaluation of speed and strength in this sport and justifies the usefulness of the laboratory test in the assessment of the actual sports performance in paracanoeists. The other reasons for using the WAnT in paracanoeists include a high level of correlations found between LA concentrations following the $200 \mathrm{~m}$ on water race and the WAnT $(\mathrm{R}=0.913)$, which confirms a similar exercise metabolism of these tasks (Figure 2). Furthermore, a graphical analysis of the relationships between the differences and the mean value from the measurements demonstrated that the values were within the 95\% confidence interval (Figure 4), and had a trivial bias. 


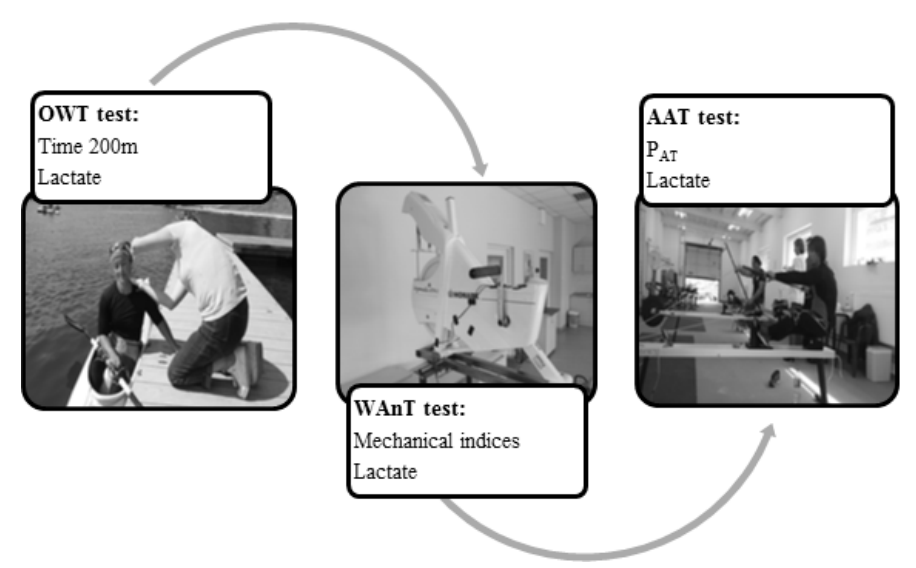

Figure 1

Study design and testing sequence. OWT - on-water test, WAnT - Wingate anaerobic test, AAT - anaerobic threshold test, $P_{A T}$ - power at anaerobic threshold

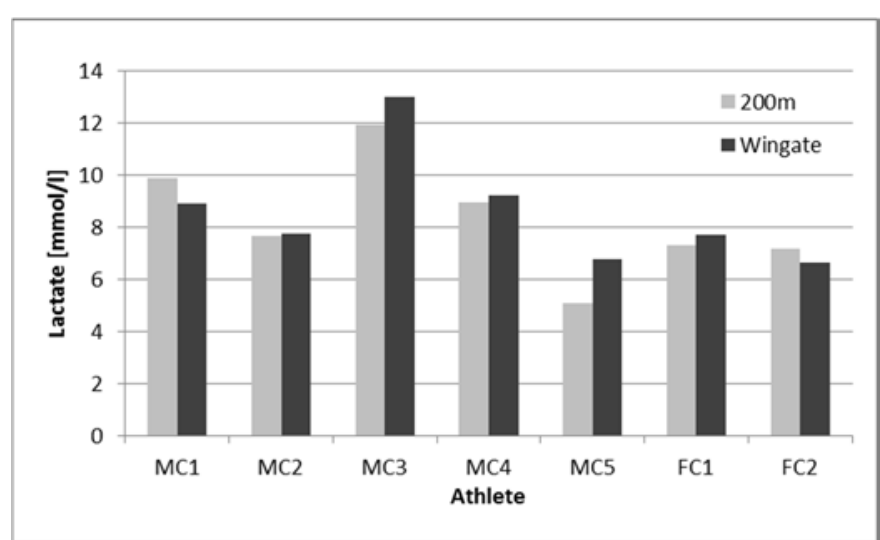

Figure 2

Individual comparison of blood lactate levels in paracanoeists tested after the $200 \mathrm{~m}$ race on water and after the Wingate test $(n=7)$. MC - male canoeist, FC-female canoeist

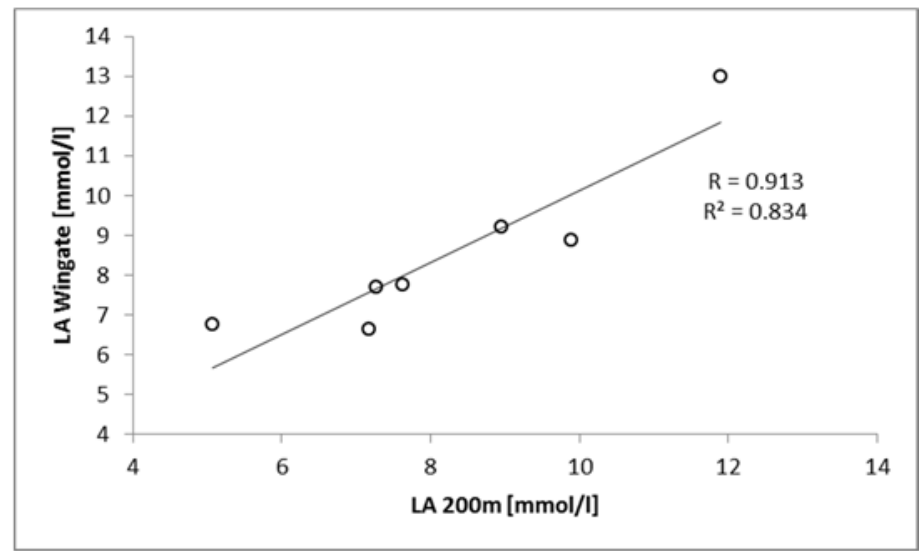

Figure 3

Correlation between lactate (LA) concentration after a $200 \mathrm{~m}$ race on water and after the Wingate test in the group of paracanoeists $(n=7)$ 


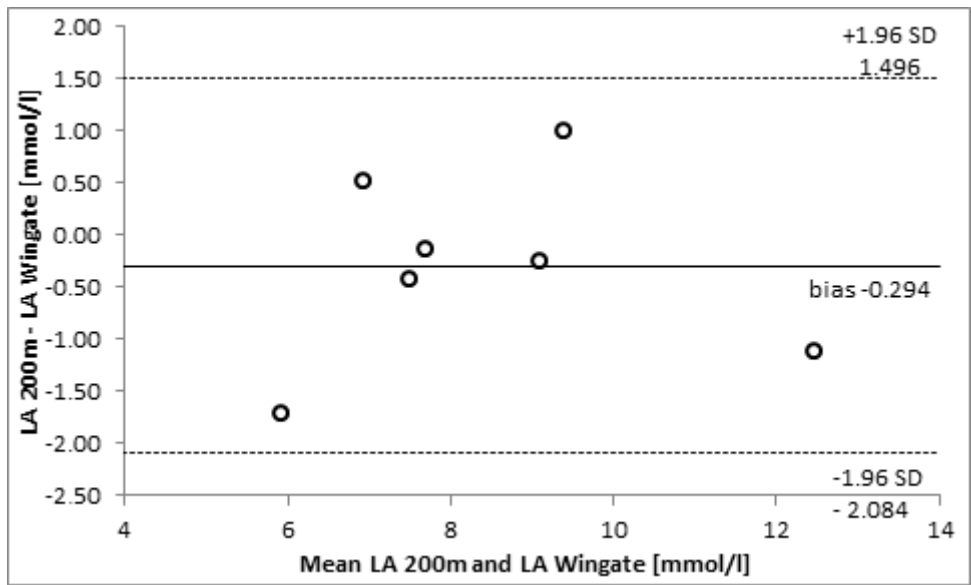

\section{Figure 4}

Bland-Altman plot: dashed lines indicate the 95\% limit of agreement, while continuous lines indicate mean difference (bias) in blood lactate levels (LA) after the

$200 m$ race and after the Wingate test in the group of paracanoeists $(n=7)$

\section{Table 1}

Results of Pearson's correlation between the $200 \mathrm{~m}$ time (on-water test) and mechanical indices obtained in the Wingate test and power at the anaerobic threshold in the group of paracanoeists $(n=7)$.

\begin{tabular}{llllllll}
\hline \multirow{2}{*}{ Test/Variable } & Unit & mean $\pm \mathrm{SD}$ & $\min$ & $\max$ & \multicolumn{3}{c}{ Correlation with $200 \mathrm{~m}$ time } \\
& & & & $\mathrm{R}$ & $p$ & effect size \\
\hline
\end{tabular}

\section{On-Water Test}

$200 \mathrm{~m}$ time $\quad[\mathrm{s}] \quad 58.8 \pm 10.7 \quad 43.6 \quad 60.1$

\section{Wingate test}

$\begin{array}{lccccccc}\mathrm{W} & {[\mathrm{J} / \mathrm{kg}]} & 166 \pm 43,5 & 135 & 245 & -0.882 & 0.009^{*} & \text { Very strong } \\ \text { Pmean } & {[\mathrm{W} / \mathrm{kg}]} & 5.54 \pm 1.45 & 4.49 & 8.19 & -0.882 & 0.009^{*} & \text { Very strong } \\ P_{\text {peak }} & {[\mathrm{W} / \mathrm{kg}]} & 7.08 \pm 1.93 & 5.31 & 10.5 & -0.796 & 0.032^{*} & \text { Very strong } \\ \mathrm{T}_{\mathrm{tp}} & {[\mathrm{s}]} & 2.69 \pm 0.74 & 1.97 & 3.50 & 0.593 & 0.160^{\mathrm{ns}} & \text { Strong } \\ \mathrm{T}_{\mathrm{kp}} & {[\mathrm{s}]} & 3.04 \pm 1.01 & 1.98 & 4.88 & 0.516 & 0.236^{\mathrm{ns}} & \text { Strong } \\ \mathrm{FF} & {[\mathrm{W} / \mathrm{s}]} & 10.5 \pm 5.43 & 6.64 & 21.0 & -0.494 & 0.260^{\mathrm{ns}} & \text { Medium }\end{array}$

Anaerobic threshold test

$\begin{array}{llllllll}\mathrm{P}_{\mathrm{AT}} & {[\mathrm{W} / \mathrm{kg}]} & 1.16 \pm 0.51 & 0.74 & 1.96 & -0.921 & 0.001^{*} & \text { Very strong }\end{array}$

$W$ - total work, $P_{\text {mean }}$ - mean power, $P_{\text {peak }}$ - peak power, $T_{t p}$ - time to reach peak power, $T_{k p}$ - time of maintaining peak power, FF-power drop index, $P_{A T-}$ power at anaerobic threshold, *statistically significant correlations, ns-non-significant 
It should be emphasized that the choice of tests for the evaluation of anaerobic capacity was based on the experiences gathered among the able-bodied athletes and adapted to the functional capabilities of disabled athletes (Byrnes and Kearney, 1997; Sitkowski, 2002). Numerous studies (Sitkowski and Grucza, 2009; Sitkowski, 2002; Someren and Palmer, 2003; Starczewski and Molik, 2014; Van Someren and Howatson, 2008) have confirmed the validity of using the Wingate test for canoeists, especially in those competing in $200 \mathrm{~m}$ races. Bearing this in mind, the test procedure was adjusted by selecting the load resulting from the classifications and sex of the athletes, as described in a study by Starczewski et al. (2014).

Disabled athletes have attracted much attention from researchers from different fields of science and many tests and exercises used previously in able-bodied athletes have been adapted for the diagnostic purposes of disabled athletes (Gołaś et al., 2017), thus the dedicated validation studies are needed. To date, the WAnT has been used to evaluate the validity of the functional classification of athletes of various disabled sports (Marszalek et al., 2015; Morgulec-Adamowicz et al., 2011; Prokopowicz et al., 2017; Starczewski and Molik, 2014). The use of the WAnT to evaluate the performance level of disabled athletes was negligible (Marszalek et al., 2015). Correlations between the $200 \mathrm{~m}$ race time and the values of the indices obtained in the WAnT confirm the significant relationships between the selected anaerobic capacity indices and sports performance (Table 1). These results are consistent with the results obtained in the group of able-bodied canoeists who were medal winners in World Championships. In a study by Sitkowski (2002) it was indicated that the anaerobic capacity indices as determinants of sports performance in the group of adult athletes included total work in the test and peak power expressed relative to body weight. In our study, significant correlations between the sports performance and the WAnT results were also found in W, Ppeak, and Pmean. Another study with British canoeists found significant correlations between the $200 \mathrm{~m}$ race results and the total work in the WAnT (Someren and Palmer, 2003). Similar observations to those in the abovementioned studies of paracanoeists were made in research of 11 female and 12 male Japanese canoeists. A strong correlation of mean and peak power with sports performance was observed in a study by Hamano et al. (1998). Taking into account the results achieved in various study groups and in our study, it can be concluded that indices measured in the WAnT which significantly correlated with sports performance, are similar in the groups of canoeists and paracanoeists, which confirms the validity of this test.

Other directions of research on indices determining sports performance in paracanoeing including indices determining aerobic capacity, were found. In our study, the highest values of the correlation coefficients were recorded between the time of the $200 \mathrm{~m}$ race and the relative power at the anaerobic threshold $(R=$ $0.921, p=0.001)$. The relationship obtained may be surprising due to a relatively low percentage of aerobic metabolism (ca. 37\%) in the total energy demand in the $200 \mathrm{~m}$ competitive effort (Byrnes and Kearn, 1997; Zamparo, 1999). However, in the study of Van Someren and Howatson (2008) it was found that power output at lactate turnpoint (LTP) corresponding to AT showed significant correlations with the results of $200 \mathrm{~m}$ and $500 \mathrm{~m}$ races. Those authors suggested that $200 \mathrm{~m}$ time could be predicted based on the chest girth, proximal humerus breadth, peak power, total work and the fatigue index in the $30 \mathrm{~s}$ test on a canoe ergometer with a standard error of only $1.7 \%$.

The main factor which could have affected the conclusion of the study is the small sample size. However, we made every effort to engage the maximum number of elite paracanoeists by carrying out measurements during one of the most important competitions of the season. On the other hand, our participants can be considered representative due to their experience in competitions and a high sports level. Another limitation of the study is that the ATT was only used to establish AT indices in relation to LA, and no $\mathrm{VO}_{2}$ or maximal aerobic indices were measured. This aspect should be considered in future studies.

The presented WAnT and ATT protocols are usefull in assessment of anaerobic and aerobic capacity in paracanoeists. The analyses suggest that the results of the WAnT mechanical 
variables and ATT may be used in determining the performance level of these athletes. The above-mentioned test protocols seem to be useful tools for coaches in assessing power and strength of paralympic athletes.

In conclusion, the research confirms high validity of the WAnT in paracanoeing. It is an accurate tool for assessment of disabled canoers which aim to increase their sports performance. The study also indicates that values of $\mathrm{W}, \mathrm{P}_{\text {mean, }}$ and $P_{\text {peak }}$ in the WAnT and $\mathrm{P}_{\mathrm{AT}}$ in the ATT can be used as performance predictors of the paralympic $200 \mathrm{~m}$ race.

\section{References}

Bishop, D. (2000). Physiological predictors of flat-water kayak performance in women. European Journal of Applied Physiology, 82, 91-97. https://doi.org/10.1007/s004210050656

Byrnes, W. C., \& Kearney, J. T. (1997). Aerobic and anaerobic contributions during simulated canoe/kayak sprint events 1256. Medicine and Science in Sports and Exercise, 29(5), 220.

Dotan, R., \& Bar-Or, O. (1983). Load optimization for the Wingate anaerobic test. European Journal of Applied Physiology and Occupational Physiology, 51(3), 409-417. https://doi.org/ 10.1007/BF00429077

Gołaś, A., Zwierzchowska, A., Maszczyk, A., Wilk, M., Stastny, P., \& Zając A. (2017) Journal of Human Kinetics, 60, 209-215. https://doi.org/10.1515/hukin-2017-0110.

Hamano, S., Ochi, E., Tsuchiya, Y., Muramatsu, E., Suzukawa, K., \& Igawa, S. (2015). Relationship between performance test and body composition/physical strength characteristic in sprint canoe and kayak paddlers. Open Access Journal of Sports Medicine, 6, 191-195. https://doi.org/10.2147/OAJSM.S82295

Hutzler, Y., Ochana, S., Bolotin, R., \& Kalina, E. (1998). Aerobic and anaerobic arm-cranking power outputs of males with lower limb impairments: relationship with sport participation intensity, age, impairment and functional classification. Spinal Cord 36(3), 205-212. https://doi.org/10.1038/sj.sc.3100627

Hübner-Wozniak, E., Kosmol, A., Lutoslawska, G., \& Bem, E. Z. (2004). Anaerobic performance of arms and legs in male and female free style wrestlers. Journal of Science and Medicine in Sport, 7(4), 473480. https://doi.org/10.1016/S1440-2440(04)80266-4

International Canoe Federation, Paracanoe Competition Rules 2019. [2019, January 15]. Retrieved July 15, 2020, from https://www.canoeicf.com/sites/default/files/rules_paracanoe_2019.pdf

Marszalek, J., Molik, B., Gomez, M. A., Skučas, K., Lencse-Mucha, J., Rekowski, W., Vaida, P., Rutkowska, I., \& Kaźmierska-Kowalewska K. (2015). Relationships between anaerobic performance, field tests and game performance of sitting volleyball players. Journal of Human Kinetics, 48(1), 25-32. https://doi.org/10.1515/hukin-2015-0088.

Morgulec-Adamowicz, N., Kosmol, A., Molik, B., Yilla, A. B., \& Laskin, J. J. (2011). Aerobic, anaerobic, and skill performance with regard to classification in wheelchair rugby athletes. Research Quarterly for Exercise and Sport, 82(1), 61-69. https://doi.org/10.1080/02701367.2011.10599722

Prokopowicz, G., Molik, B., Prokopowicz, K., Ogonowska-Słodownik, A., Lencse-Mucha, J., MorgulecAdamowicz, N., Kosmol, A., Perkowski, K., Chamera, T., \& Grzywacz, T. (2017). Anaerobic Capacity of Sailors with Disabilities. Polish Journal of Sport Tourism, 24(1), 21-24. https://doi.org/10.1515/pjst-2017-0003

Rosenthal, J. A. (1996). Qualitative descriptors of strength of association and effect size. Journal of Social Service Research, 21(4), 37-59. https://doi.org/10.1300/J079v21n04_02

Sitkowski, D., \& Grucza, R. (2009). Age-related changes and gender differences of upper body anaerobic performance in male and female sprint kayakers. Biology of Sport, 26(4), 325.

Sitkowski, D. (2002). Some indices distinguishing Olympic or world championship medallists in sprint kayaking. Biology of Sport, 19(2), 133-148.

Someren, K. V., \& Palmer, G. S. (2003). Prediction of 200-m sprint kayaking performance. Canadian Journal of Applied Physiology, 28(4), 505-517. https://doi.org/10.1139/h03-039

Starczewski, M., \& Molik, B. (2014). Selected anaerobic capacity parameters regard to paracanoe functional classification. Advances in Rehabilitation, 28(1), 31. https://doi.org/10.2478/rehab-2014-0031 
Starczewski, M. (2013). Parakajakarstwo-nowy sport na igrzyskach paraolimpijskich. Advances in Rehabilitation, 27(1), 63-8. https://doi.org/10.2478/rehab-2014-0007

Van Someren, K. A., \& Howatson, G. (2008). Prediction of flatwater kayaking performance. International Journal of Sports Physiology and Performance, 3, 207-218. https://doi.org/10.1123/ijspp.3.2.207

Zamparo, P., Capelli, C., \& Guerrini, G. (1999). Energetics of kayaking at submaximal and maximal speeds. European Journal of Applied Physiology and Occupational Physiology, 80(6), 542-548. https://doi.org/10.1007/s004210050632

\section{Corresponding author:}

\section{Piotr Zmijewski}

Jozef Pilsudski University of Physical Education in Warsaw, Warsaw, Poland Marymoncka 34, 00-809 Warszawa

E-mail: zmijewski@op.pl 